Short Paper

\title{
Gründüngung mittels Wintereinsaat in Weinbergen mit starkem vegetativem Wachstum
}

\section{Green manuring with winter crops in vineyards with intensive vegetative growth}

\section{Sovescio invernale in vigneti ad alta vigoria}

\section{Florian Haas ${ }^{1}$, Julia Martinelli ${ }^{1}$, Barbara Raifer ${ }^{1}$}

${ }^{1}$ Versuchszentrum Laimburg, Auer, Italien

\section{ABSTRACT}

Green manuring is already well known as a technique to rise the soil fertility in viticulture. The bare soil tillage, as well as the utilization of legumes in the seed mixture of the green manure plants has a strong impact on mineralization of nitrogen. These facts reduced the application of green manuring on vineyards with low vegetative growth. The utilization of nitrogen absorbing cereals as alternative for a green manure in high vigor vineyards is analyzed in the present research trial. These plants should raise the soil organic carbon and absorb the mineral nitrogen released as a result of soil tillage. This should avoid a further increase of vegetative growth by the vines.

\section{KEYWORDS}

Green manuring, cover crops, cereals, soil organic matter, viticulture, high vigour

\section{CITE ARTICLE AS}

Haas Florian, Martinelli Julia, Raifer Barbara et.al. (2021). Green manuring with winter crops in vineyards with intensive vegetative growth. Laimburg Journal 03/2021 DOI: $10.23796 /$ LJ/2021.006

\section{CORRESPONDING AUTHOR}

Florian Haas

Laimburg 6, Pfatten, 39040 Auer (BZ), Italien

florian.haas@laimburg.it +390417969613 


\section{EINLEITUNG}

Während des vergangenen Jahrzehntes entwickelte sich in der EU-Agrarpolitik die Ökologisierung und der Aufbau einer nachhaltigen Landwirtschaft immer mehr zum Kernthema. Der Boden spielt dabei die grundlegende Rolle [1]. Doch zunehmende Urbanisierung, hochintensive Landwirtschaft, tiefe und häufige Bodenbearbeitung, offener Boden sowie dauerhafter Einsatz von Mineraldünger führen zu starkem $\mathrm{Ab}$ bau der organischen Substanz im Boden und somit zum Verlust der Bodenfruchtbarkeit. Die EU-Kommission hat daher als die wichtigsten Prozesse, die zur Verschlechterung der Bodenqualität in der EU beitragen, die Erosion, den sinkenden Gehalt an organischen Stoffen, Verschmutzung, Versalzung, Verdichtung, Verarmung der biologischen Vielfalt der Böden, Versiegelung sowie Überschwemmungen und Erdrutsche erkannt. Gesunde Böden sind von entscheidender Bedeutung, damit die Klima- und Biodiversitätsziele im Rahmen des europäischen Grünen Deals erreicht werden können [2].

Die Strategie „Vom Hof auf den Tisch“ sieht unter anderem die Verringerung des Einsatzes und des Risikos von Pestiziden um 50\% und eine Verringerung des Einsatzes von Düngemitteln um mindestens $20 \%$ [3] vor. Dem hat nun auch das Konsortium Südtiroler Wein mit der Südtirol Wein Agenda 2030 Folge geleistet und Schritte zur Unterbindung des Einsatzes von Herbiziden und mineralischem Sticksoff in den Südtiroler Rebflächen gesetzt.

Die Folgen eines Weinanbaus mit Dauerbegrünung und ohne Herbizide wurden bereits öfters untersucht und ergaben auch einige positive Aspekte, wie eine Verringerung von überschüssigem vegetativem Wachstum [4]. Jedoch ist dies nicht immer nur ein Vorteil. In Südtirol wurde bereits beobachtet, dass normal wachsende Anlagen auch mit nur mäßigen Ertragszielen nach mehreren Jahren ohne Düngung und der Anwendung von Herbiziden im Unterstockbereich die potenzielle Fruchtbarkeit nicht halten konnten. Die gemulchte Dauerbegrünung der Fahrgasse hat in Südtirol inzwischen eine über 40 Jahre lange Tradition. Dies führt zu einer sehr mächtigen Ansammlung von organischer Substanz in den obersten $10 \mathrm{~cm}$ unter der Grasnarbe, während in tieferen Bodenschichten meist nur noch wenig Humus vorzufinden ist [5]. Die Vorteile einer gleichmäßigen Verteilung des Humusgehaltes in tieferen Bodenschichten bleiben somit aus.

Für den Südtiroler Winzer bedeutet dies nun, dass er sich in naher Zukunft neue Wege zur Erhaltung und zum Aufbau der Bodenfruchtbarkeit zurechtlegen muss. Einer dieser Wege kann sicherlich die bereits bekannte Gründüngung mittels Einsaat winterharter Pflanzen während der Ruhephase der Rebe nach der Lese sein. Dies ist keine neue Technik, sondern war seit der Zeit der Römer bis hin zur Mitte des vergangenen Jahrhunderts normale Praxis, die neuerdings wieder die Aufmerksamkeit der Winzer und der Forschung weckt [6] [7] [8]. Das VZ Laimburg hat ebenso bereits 2009 mit Versuchen zu diesem Thema begonnen. Dabei ging es darum, in einer sehr schwach wachsenden Rebanlage mit niedrigem Gehalt an organischer Substanz die Bodenfruchtbarkeit zu steigern und dadurch die Traubenqualität zu verbessern. Diese Erwartungen konnten durch die vorgestellten Maßnahmen klar erfüllt werden [5]. Die Anwendung der Wintereinsaat zur Gründüngung in schwachen Anlagen konnte sich inzwischen sehr gut in der Praxis etablieren.

Doch wie sieht es mit der Anwendung einer Wintergründüngung in eher stark wachsenden Lagen aus?

Die notwendige mechanische Einarbeitung der Begrünung zur Saatbett-Vorbereitung fördert die Mineralisation der organischen Substanz durch die Belüftung und die Durchmischung der obersten Bodenschichten. Die Mineralisation kann Nährstoffe freisetzen und zur Belebung der Rebanlage beitragen [9] [10]. Dies ist mitunter ein Grund dafür, dass Südtiroler Winzer vor einer Wintergründüngung in stark wachsenden Anlagen noch zurückschrecken.

Stickstoffzehrende Pflanzen, wie es verschiedene Getreidesorten sind, könnten den anfänglichen Stickstoffschub aufnehmen und in ihrer Biomasse einschließen [11]. Auf diese Weise könnte ein zusätzlicher vegetativer Wachstumsschub in einer bereits stark wachsenden Rebanlage vermieden werden. Ob Roggen, Weizen, Dinkel oder Gerste für diesen Zweck geeignet sind und welche Auswirkung das alleinige mechanische Einarbeiten der Grasnarbe auf Boden und Rebe hat, sollte daher in einem 7-jährigen Gründüngungsversuch am VZ Laimburg untersucht werden.
In diesem Artikel werden nun Ergebnisse eines Versuches zur Gründüngung in stark wachsenden Rebanlagen, welcher in zwei Anlagen in Kaltern und Meran durchgeführt wurde, vorgestellt. Ziel dieser Arbeit war es, aufzuzeigen, ob und welche Getreidearten den Stickstoffschub der Bodenbearbeitung auffangen und in der Folge einen vegetativen Wachstumsschub der Reben verhindern können.

\section{METHODEN}

Der Versuch wurde im Zeitraum 2014-2020 in zwei Versuchsanlagen des VZ Laimburg durchgeführt. Von 2014-2018 befand sich der Versuch in einer Anlage in Kaltern mit der Sorte Goldmuskateller (Pflanzjahr 2002) und ab 2017 in einer Gewürztraminer-Anlage (Pflanzjahr 2013) in Meran. Der Versuch wurde von Kaltern nach Meran verlegt, da es durch die topographische Lage in manchen Jahren zu einer nicht ausreichenden Auflaufrate der Gründüngungspflanzen, sowie zu phytopathologischen Problemen an den Trauben kam. Aus diesen Gründen konnten nicht alle Auswertungen in allen Versuchsjahren durchgeführt werden.

Die in Südtirol übliche Fahrgasse mit einer niedrigen Mulchmischung und mehrmaligen Mulchschnitten je nach Witterungssituation stellte die Kontrollvariante dar. Dieser Kontrollvariante wurden 5 Versuchsvarianten gegenübergestellt: die oberflächliche Bodenbearbeitung zur Saatbett-Vorbereitung mittels eines Spatenpflugs bis auf maximal $10 \mathrm{~cm}$ Bodentiefe ohne jegliche Einsaat und vier Varianten mit derselben Bodenbearbeitung zur Saatbett-Vorbereitung und jeweils einer unterschiedlichen Getreideart zur Wintergründüngung. Die Getreidearten wurden unter Berücksichtigung ihres Stickstoffanspruchs und ihrer Wuchsleistung (Biomasseproduktion) ausgewählt. So benötigt z.B. der Weizen für seine Entwicklung im professionellem Kornanbau jährlich bis zu $200 \mathrm{~kg} \mathrm{~N} / \mathrm{ha}$, Roggen bis zu $170 \mathrm{~kg} \mathrm{~N} / \mathrm{ha}$, Gerste bis zu 180 kg N/ha und Dinkel 210 kg N/ha [11]. Diese Gründüngungspflanzen entwickelten sich bis kurz vor der Rebblüte, wurden dann mit einer Profilwalze gewalzt und als Bodenbedeckung auf der Bodenoberfläche belassen.

Um die Auswirkungen der Varianten auf den Nährstoffhaushalt der Anlage und die Wüchsigkeit der Reben bewerten zu können, wurde die Wuchshöhe der Einsaatpflanzen gemessen, Bodenanalysen des Oberbodens $(0-30 \mathrm{~cm})$ und des Unterbodens $(30-60 \mathrm{~cm})$ 
durchgeführt, sowie Rebblätter und Beeren auf ihren Nährstoffgehalt untersucht. Die Beeren der Trauben wurden durch die Analyse ihres Mostes (120 Beeren/Probe) kurz vor der Ernte auch auf ihre qualitätsgebenden Eigenschaften wie der Gehalt an Zucker ( $\left.{ }^{\circ} \mathrm{KMW}\right)$, Säuren (titrierbare Gesamtsäure), Weinsäure $(\mathrm{g} / \mathrm{L})$ und Apfelsäure $(\mathrm{g} / \mathrm{L}), \mathrm{pH}$ Wert und hefeverwertbarer Stickstoff (HVS in $\mathrm{mg} / \mathrm{L}$ ) mittels einer spektroskopischen Analysemethode (FTIR WineScan ${ }^{\text {TM }}$, FOSS, Denmark) untersucht. Die generative und vegetative Produktionsleistung der Reben wurde ermittelt und daraus der Ravaz-Index errechnet [12].

\section{ERGEBNISSE}

Die erste Bodenbearbeitung nach der Lese 2014 bewirkte in der Goldmuskateller Anlage in Kaltern einen deutlichen Anstieg des Stickstoffgehaltes in der Bodenlösung einen Monat nach erfolgter Bodenbearbeitung (Abb. 1). Jene Parzellen, in welchen sich schon die Keimlinge der Getreidesorten entwickelt hatten, konnten jedoch nicht von der unbearbeiteten Kontrolle unterschieden werden. Der hohe Stickstoffgehalt der reinen Bodenbearbeitungsvariante konnte beim Probetermin im darauffolgenden Frühling nicht mehr erkannt werden.

Vergleicht man die mineralischen Stickstoffgehalte im Dezember bei den Bewirtschaftungsarten, also Dauerbegrünung, Bodenbearbeitung ohne Einsaat und Wintergründüngung im Mittelwert aller Getreidesorten, ergibt sich ein klares Bild, wie in Abb. 2 (links) abgebildet. Die Keimlinge der Getreidesorten konnten den Stickstoffschub verwerten. Dieselbe Analyse wurde im Juni des darauffolgenden Jahres 2015 wiederholt. Dabei ging klar hervor, dass der freie Stickstoff vom Vorjahr bei der Bodenbearbeitungsvariante ohne Einsaat in den Wintermonaten ausgewaschen wurde, da sich bis zur Probenahme im Frühlin keine neue Krautschicht gebiltet hatte ist Abb. 2 (rechts).

Einen ähnlichen Verlauf konnte im Herbst des Folgejahres 2015 in Abb. 3 (blaue Balken) festgestellt werden. Hierbei ist wiederum ein starker Anstieg des verfügbaren Stickstoffgehaltes im Boden nach der Bodenbearbeitung zu beobachten. Bei dieser Analyse erkennt man auch einen Anstieg des verfügbaren Stickstoffs bei den Wintergründüngungsvarianten mit den Getreidesorten. Im Sommer des Jahres 2016 (grüner Balken) konnte hingegen kein Unterschied zwischen den bearbeiteten Varianten und der Dauerbegrünung gemessen werden.

Beim Parameter organische Trockensubstanz im Boden konnte von 2014-2018 eine tendenzielle Zunahme bei den Einsaatvarianten festgestellt werden. Hierbei beschränkte sich die Auswirkung der Einsaaten nur auf den Oberboden (Abb. 4). Die Zunahme der organischen Trockensubstanz war in den ersten $30 \mathrm{~cm}$ Bodentiefe als deutliche Tendenz ersichtlich, auch wenn diese durch die hohe hohe Streuung der Messwerte nicht statistisch signifikant war.

Im Unterboden hingegen konnte keine Auswirkung der Getreideeinsaaten auf den Humusgehalt im Laufe der 4 Versuchsjahre festgestellt werden (Abb. 5).

Auf das Beerengewicht und den Zuckeroder Säuregehalt nach den vier Versuchsjahren hatten die Bodenbearbeitungs- und die Einsaatvarianten keinen signifikanten Einfluss. Nur der hefeverwertbare Stickstoffgehalt (HVS) des Goldmuskatellers hat schon von Anfang an etwas auf die veränderte Stickstoffverfügbarkeit im Boden reagiert. So war beispielsweise bereits im ersten Versuchsjahr 2015 ein tendenziell höherer HVSGehalt in den Trauben der Variante "mechanische Bodenbearbeitung" zu erkennen (Abb. 6, links). Nach vier Versuchsjahren war diese Tendenz immer noch ersichtlich (Abb. 6, rechts). Die Erklärung hierfür liegt dabei in der verringerten Stickstoffkonkurrenz von Seiten der fehlenden Begrünung im Fühjahr gegenüber den Reben. Die erhöhte Stickstoffverfügbarkeit während der Wintermonate infolge der Bodenbearbeitung für die Saatbettbereitung ist dafür nicht aussschlaggebend, da sich die Rebe im Winter in Winteruhe befindet daher keinen Stickstoff aufnimmt.

Zwischen den Getreidearten gab es im Wuchsverhalten einige messbare Unterschiede. So konnte beim Roggen eine stets signifikant höhere Stängelentwicklung festgestellt werden (Abb. 7). Die Wuchshöhe von Gerste, Weizen und Dinkel war hingegen immer vergleichbar auf niederem $\mathrm{Ni}$ veau. Durch die höhere Wuchsöhe des Roggens kann auch auf eine größere Biomasse im Wurzelbereich geschlossen werden. Dies bringt somit einen schnelleren Humusaufbau und eine bessere Stickstoffverwertung mit sich.

Ab dem Jahre 2017 wurde dieselbe Versuchsmethodik am Standort Moarhof in Labers bei Meran wiederholt. In der
Gewürztraminer -Anlage konnte kein signifikanter Unterschied im verfügbaren Stickstoffgehalt im Boden nach der Bodenbearbeitung gemessen werden. Dies deutet darauf hin, dass in dieser Anlage die Stickstofffreisetzung durch die Störung der Grasnarbe geringer war als in der Goldmuskateller-Anlage (Abb. 8, links). Die Grafik zeigt deutlich, dass die Kontrollvariante im Oberboden mehr freien Stickstoff enthielt als im Unterboden. Bei allen Varianten mit Bodenbearbeitung im Herbst des vorangegangenen Jahres erkennt man einen deutlich angestiegenen Stickstoffwert im Unterboden. Mit Ausnahme der Variante Dinkel war im Unterboden sogar mehr Stickstoff vorhanden als im Oberboden.

Nach 4 Jahren der Wintereinsaat ergab sich bezüglich des mineralischen Stickstoffgehaltes des Bodens keine statistisch absicherbare Veränderung (Abb. 8, rechts). Es fällt auf, dass beim Roggen der Unterschied zwischen Oberboden und Unterboden am größten war.

Auch in der Anlage in Meran konnte sich der Roggen durch eine deutlich regelmäßigere Wuchshöhe und Auflaufrate deutlich von den weiteren Getreidearten unterscheiden. Aus den schon vorher genannten Gründen kann der Roggen als besserer Einsaatpartner für die Wintergründünung im Weinbau angesehen werden.

Im Laufe der 4 Versuchsjahre führten die unterschiedlichen Getreideeinsaaten zu keinen signifikanten Auswirkungen auf den $\mathrm{Hu}$ musgehalt des Bodens (Abb. 9).

Bei den Blattanalysen der Rebblätter konnte nach 3 Jahren der Gründüngung eine tendenziell verbesserte Nährstoffversorgung im Vergleich zur Kontrolle festgestellt werden (Abb. 10, links und rechts).

Nach der Blüte gegen Ende Mai wurde in der Anlage eine Erhebung der potenziellen Fruchtbarkeit durchgeführt. Dabei wurden die sichtbaren Gescheine je Trieb gezählt. Diese Erhebung ergab jedoch keinen signifikanten Unterschied zwischen den Versuchsvarianten.

Bei den Reifeparametern konnten in dieser Anlage in den 4 Versuchsjahren keine Unterschiede festgestellt werden.

In der Gewürztraminer-Anlage wurden in den Jahren 2019 und 2020 Ernte- und Schnittholzerhebungen durchgeführt. Beim Traubengewicht konnte eine leichte Tendenz zu etwas schwereren Trauben bei de 
Variante „mechanische Bodenbearbeitung ohne Einsaat" bemerkt werden (Abb. 11).

Diese leichte Tendenz konnte sich jedoch im Ertrag nicht bestätigen (Abb. 12).

Somit wurde die generative Produktion der Reben durch den Einsatz von Getreide als Wintereinsaat nicht beeinflusst. Auch die reine Bodenbearbeitung hat dabei nur eine leichte tendenzielle Steigerung verzeichnen können.

Das Schnittholzgewicht als Mass der vegetativen Produktion der Reben eines jeden Jahres blieb ebenso unverändert (Abb. 13).

Zusammenfassend kann festgestellt werden, dass sowohl die Gründüngungen als auch die reine Bodenbearbeitung zu keiner Änderung des Verhältnisses zwischen der generativen- und vegetativen Produktion (Ravaz-Index) führten (Abb. 14).

\section{DISKUSSION}

Der freie Stickstoffgehalt (N-min) im Boden wurde zum Teil durch die Bodenbearbeitung leicht erhöht. Diese bessere Stickstoffverfügbarkeit nach einer Bodenbearbeitung ist sehr typisch für Böden, welche nach vielen Jahren erstmals wieder bearbeitet werden [13] [14]. Überraschend war jedoch, dass die kleinen Keimlinge der Herbsteinsaat in manchen Probenahmen einem Monat nach der Einsaat schon einen signifikant geringeren Gehalt an verfügbarem Stickstoff im Boden aufzeigen konnten und somit diesen freien Stickstoff für sich beanspruchen konnten. Daher ist es wichtig, nach der mechanischen Bearbeitung des Bodens möglichst schnell den Anwuchs der eingesäten Pflanzen zu erreichen, um Stickstoffverluste zu minimieren. Es sollte also auch nicht zu spät eingesät werden, damit ein rascher Aufwuchs erfolgen kann und der Boden nicht über den Winter offenbleibt. In der Gewürztraminer-Anlage in Meran war der Unterschied beim freien Stickstoffgehalt zwischen den Bodenbearbeitungsvarianten und der Kontrolle geringer als in Kaltern. Dies kann darauf zurückgeführt werden, dass die Anlage in Meran auch deutlich jünger war und somit in den obersten Bodenschichten die Ansammlung von organischer Substanz aus der Grasnarbe noch nicht so mächtig war wie in der älteren Goldmuskateller-Anlage.

Der Gehalt an organischer Trockensubstanz konnte durch die Einsaat von Getreidesorten in beiden Versuchsanlagen und nach jeweils vier aufeinanderfolgenden Einsaaten

\section{ZUSAMMENFASSUNG}

Es gibt bereits eine Vielzahl von Forschungsarbeiten zum Einsatz der Gründünung zur Förderung der Bodenfruchtbarkeit im Weinbau. Die Bodenbearbeitung selbst gibt dabei einen starken Impuls zur Mineralisierung des Stickstoffs im Boden, wie auch Leguminosen in der Einsaatmischung. Dies beschränkt den Einsatz auf Rebanlagen mit schwachem vegetativen Wachstum. Der Einsatz von stickstoffzehrenden Getreidearten wird als mögliche Alternative für die Gründüngung in Rebanlagen mit starkem vegetativen Wachstum in dieser Forschungsarbeit untersucht. Diese Pflanzen sollen die Humusverteilung im Boden verbessern und den durch die Saatbettbereitung freiwerdenden Stickstoff binden und ein stärkeres Wachstum der Reben vermeiden.

\section{RIASSUNTO}

L'utilizzo del sovescio come tecnica per migliorare la fertilità del suolo viticolo è già una tecnica ben affermata. La lavorazione del terreno per la preparazione del letto di semina da un forte impulso alla mineralizzazione dell'azoto e quindi alla sua disponibilità, come lo fanno anche le leguminose se fanno parte del mescuglio per il sovescio. Questi fatti hanno fino ad ora limitato I'applicazione dei sovesci ai siti con bassa vigoria vegetativa. Per poter proporre una variante del sovescio anche per siti più vigorosi si esamina l'utilizzo di cereali con forte richiesta di azoto come piante da sovescio. Queste piante hanno lo scopo da una parte di aumentare la sostanza organica nel suolo e dall'altra di metabolizzare l'azoto liberatosi dalla lavorazione del terreno per la preparazione del letto di semina e quindi prevenire un ulteriore aumento della intensità di crescita vegetativa della vite.

nicht gesteigert werden. Eine Erklärung hierfür kann in der bereits hohen Bodenfruchtbarkeit der ausgewählten Versuchsanlagen gefunden werden, welche auch bereits vor Versuchsbeginn recht hohe Humusgehalte aufwiesen. In Kaltern war im Oberboden schon 2,5 bis knapp unter $3 \%$ organische Trockensubstanz vorzufinden, während in Meran sogar Gehalte zwischen 3,5 und 4\% gemessen werden konnten. Dadurch ist auch die Wahrscheinlichkeit einer weiteren Steigerung dieser schon beachtlichen $\mathrm{Hu}$ musgehalte eher gering [15]. Vielleicht wurde durch die Einsaaten aber auch einfach wenig organische Masse erzeugt, oder die erzeugte organische Masse wurde durch häufiges und eher tiefes Mulchen während der Vegetationsperiode schnell wieder abgebaut. Ebenso könnte eine zu tiefe Bearbeitung bei der Saatbettbereitung eine Rolle gespielt haben. Dieser Punkt bedarf weiterer Klärung, denn warum sollte man Einsaaten durchführen, wenn sich keine anhaltende Bodenverbesserung ergibt? Wo liegen die Grenzen der Humusanreicherung bei den typischen, skelettreichen Südtiroler
Weinbauböden? Und müsste nicht auch in den Unterböden noch Raum für einen weiteren Humusaufbau sein? Neben interessanten Erkenntnissen, lässt auch dieser Versuch noch einige Fragen offen.

Der trotz allem invasive Eingriff einer Bodenbearbeitung in ein recht stabiles Dauerbegrünungs-Ökosystem in der Fahrgasse hat in diesem Versuch nur minimalen Einfluss auf die Qualitätseigenschaften der Trauben zeigen können. Einzig der Stickstoffgehalt der Blätter und der Beeren konnte in einzelnen Jahren bei den Einsaatvarianten leicht gesteigert werden. In anderen Versuchen konnten indessen leicht höhere Zucker- und niedrigere Säuregehalte [16] und deutlich höhere Stickstoffgehalte [17] [5] [7] in den Gründüngungsvarianten festgestellt werden. In diesen Studien wurden jedoch auch Leguminosen in den Saatmischungen verwendet, welche einen verbesserten Stickstoffeintrag in den Boden und weitaus tiefere Durchwurzelungstiefen als das Getreide mit sich bringen [18]. 
Ebenso verhielt es sich mit den Ertragsleistungen bei der Gewürztraminer-Anlage. Sowohl die Gründüngung mit den Getreidepflanzen als auch die reine Bodenbearbeitung konnten keine weitere Steigerung des vegetativen Wachstums nach sich ziehen. Sowohl die generative Leistung bei der Fruchtbarkeitserhebung als auch beim Erntegewicht zeigten keinerlei Unterschied. Dies ist auch gut nachvollziehbar, da keine wesentliche Veränderung der Bodeneigenschaften und somit der Nährstoffverfügbarkeit festzustellen war.
Als leistungsstärkster und verlässlichster Einsaatpartner für die Wintergründünung mit Getreide hat sich der Roggen erwiesen. Seine Wuchshöhe und Auflaufrate haben deutliche Vorteile gegenüber den weiteren Getreidesorten aufgezeigt.

\section{ZUSAMMENFASSUNG UND FA-} ZIT

Die Bodenbearbeitung zur Saatbettbereitung für die Herbst-Wintergründüngung kann eine zum Teil signifikant höhere Stickstofffreisetzung zur Folge haben. Die Einsaat von stickstoffzehrenden Getreidearten ist in der Lage, diese Stickstoffmenge zu verstoffwechseln und somit eine vegetative Wachstumszunahme bei den Reben zu unterbinden. Der Nährstoffhaushalt der Blätter und Beeren konnte dabei zum Teil positiv beeinflusst werden. Somit kann die Wintergründüngung mit Getreidesorten auch in Rebanlagen mit starkem vegetativem Wachstum empfohlen werden. 


\section{LITERATUR}

[1] Europäische Kommission (ed.) (2007). Faktenblatt Umwelt. Bodenschutz - eine neue Politik für die EU. Retrieved March 6, 2021, from https://ec.europa.eu/environment/archives/soil/pdf/factsheet 2007 de.pdf.

[2] Europäische Kommission (ed.) (2020). Neue Beobachtungsstelle für gesunde Böden in Europa startet. Retrieved March 6, 2021, from https://ec.europa.eu/germany/news/20201204-gesunde-boeden de.

[3] Europäische Kommission (ed.) (2020). Europas Widerstandsfähigkeit stärken. Wie wir den Verlust anbiologischer Vielfalt stoppen und ein gesundes und nachhaltiges Lebensmittelsystem aufbauen. Retrieved March 6, 2021, from https://ec.europa.eu/commission/presscorner/detail/de/ip_20_884.

[4] Celette F., Findeling A., Gary C. (2009). Competition for nitrogen in an unfertilized intercropping system Grapevine and grass cover in a Mediterranean climate. European Journal of Agronomy 30 (1), 41-51. DOI: 10.1016/j.eja.2008.07.003.

[5] Haas F., Struffi I., Raifer B. et al. (2015). Green manurin in horizontally terraced vineyards. A new approach. In: Scientific Committee of GiESCO (eds.). Actes des 19èmes Journées Internationales de Viticulture GiESCO. Pech Rouge - Montpellier, France, 31 Mai-5 Juin 2015. Groupe international $d^{\prime}$ Experts en Systèmes vitivinicoles pour la CoOpération (GiESCO), Montpellier, France, pp. 697-701.

[6] Valenti L., Pioerluigi D., Ghiglieno I. (2014). Gestione del suolo in vigneto, l'integrazione delle tecniche. L'Informatore Agrario 69 (5), 36-41.
[7] Gontier L. (2013). Engrais verts en viticulture: Incidence du type de couvert vègètal et son mode de destruction sur l'alimentation azotée de la vigne. In: Scientific Committee of GiESCO (eds.). Proceedings of the 18th International Symposium of the Group of International Experts of vitivinicultural Systems for CoOperation, Porto, Portugal, July 7-11, 2013. (Ciência e Técnica Vitivinícola : 28), pp. 299-304.

[8] Fourie, J. (2016). Soil Management in the Breede River ValleyWine Grape Region, South Africa. 4. Organic Matter and Macronutrient Content of a Medium-textured Soil. South African Journal of Enology and Viticulture 33 (1), 105-114, DOI: 10.21548/33-11312.

[9] Akbolat D., Evrendilek F., Coskan A. et al. (2009). Quantifying soil respiration in response to short-term tillage practices. A case study in southern Turkey. Acta Agriculturae Scandinavica, Section B - Soil \& Plant Science, 59 (1), 50-56, DOI: $10.1080 / 09064710701833202$.

[10] Kandeler E., Böhm K.E. (1996). Temporal dynamics of microbial biomass, xylanase activity, $\mathrm{N}$-mineralisation and potential nitrification in different tillage systems. Applied Soil Ecology 4 (3), 181-191, DOI: 10.1016/S09291393(96)00117-5.

[11] Peratoner G. (2016). Merkblätter zum Getreidebau - Projekt Regiokorn. Retrieved May 2, 2021, from http://www.laimburg.it/de/dienstleistungen/anleitungen. asp? news action=4\&news article id=538647.

[12] Ravaz, L. (1903). Sur la brunissure de la vigne. Comptes rendus hebdomadaires des séances de l'Académie des Sciences (136), 1276-1278.
[13] Bossche A.V.D., Bolle S.D., Neve S.D. et al. (2009). Effect of tillage in-tensity on $\mathrm{N}$ mineralization of different crop residues in a temperate climate. Soil and Tillage Research 103 (2), 316-324, DOI: 10.1016/j.still.2008.10.019.

[14] Thomsen I.K., Sørensen P. (2006). Tillage-induced $\mathrm{N}$ mineralization and $\mathrm{N}$ uptake in winter wheat on a coarse sandy loam. Soil and Tillage Research 89 (1), 58-69, DOI: 10.1016/j.still.2005.06.011.

[15] Barré P., Angers D.A., Basile-Doelsch I. et al. (2017). Ideas and perspectives. Can we use the soil carbon saturation deficit to quantitatively assess the soil carbon storage potential, or should we explore other strategies?. Biogeosciences Discussions [preprint], DOI: 10.5194/bg-2017-395.

[16] Rotaru L., Stoleru V., Mustea M. (2011). Fertilization with green manure on Chasselas Doré grape vine as an alternative for sustainable viticulture. Journal of Food, Agriculture and Environment 9 (3/4 part 1), 236243.

[17] Zanzotti R., Meschalchin E. (2019). Green manure effects on inorganic nitrogen dynamics in soil and its accumulation in grape must. BIO Web of Conferences 13:04010, DOI: 10.1051/bioconf/20191304010.

[18] Kutschera L., Lichtenegger E. (2010). Wurzelatlas mitteleuropäischer Ackerunkräuter und Kulturpflanzen. DLG-Verlag, Frankfurt am Main, Deutschland. 


\section{ANHANG: ABBILDUNGEN}

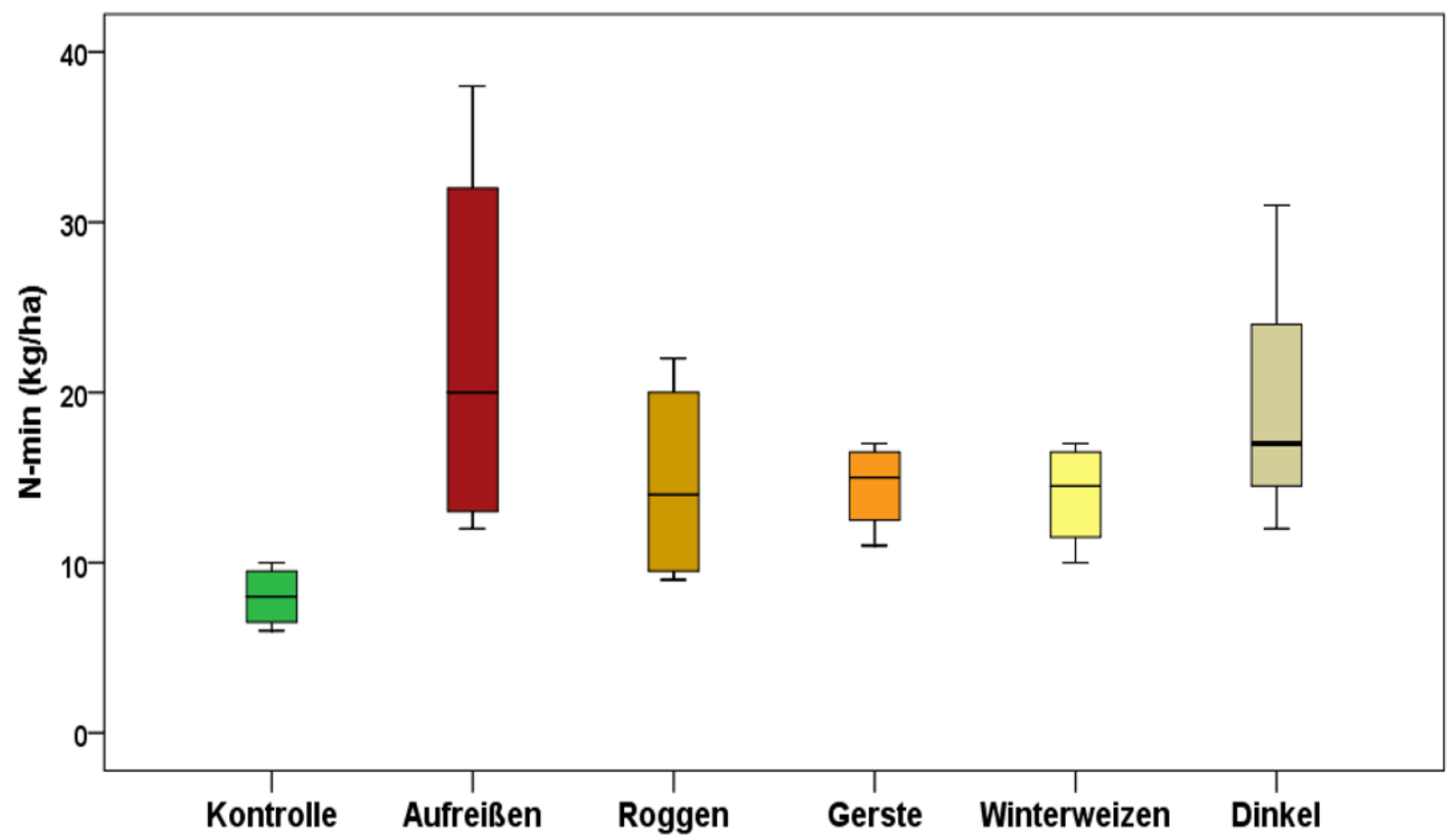

Abb. 1: N-min Gehalte vom Dezember 2014 einen Monat nach der Einsaat der Gründünungspflanzen // Plant available nitrogen from December 2014 one month after sawing of cover crops.
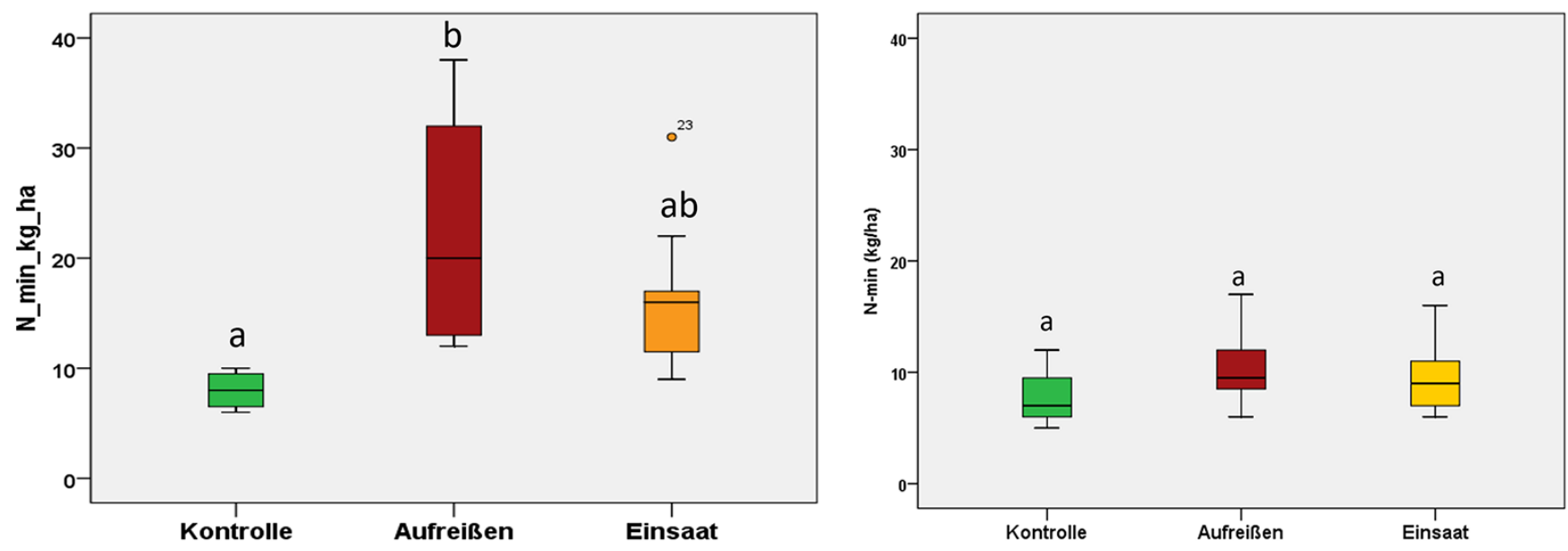

Abb. 2: N-min (kg/ha) Gehalte der Bodenbewirtschaftungsarten im Dezember 2014 (links) und im Juni 2015 (rechts); unterschiedliche Buchstaben weisen auf signifikante Unterschiede zwischen den Varianten hin (Anova mit post hoc Tukey's b HSD test p $\geq 0,05$ ). // Plant available nitrogen ( $k g / h a$ ) of the different soil management techniques from December 2014 (left) and June 2015 (right); different letters indicate means between the treatments are significantly different (Anova analysis of variance and Tuckey's bost hoc HSD test $p \geq 0,05$ ). 




Abb. 3: N-min (kg/ha) Gehalte der Bewirtschaftungsmethoden zwischen Herbst 2015 und Sommer 2016; unterschiedliche Buchstaben weisen auf signifikante Unterschiede zwischen den Varianten hin (Anova mit post hoc Tukey's b HSD test $\mathrm{p} \geq 0,05$ ). // Plant available nitrogen ( $\mathrm{kg} / \mathrm{ha}$ ) of the different soil management techniques from autumn 2015 and summer 2016; different letters indicate means between the treatments are significantly different (Anova analysis of variance and Tuckey's b post hoc HSD test $p \geq 0,05$ ).

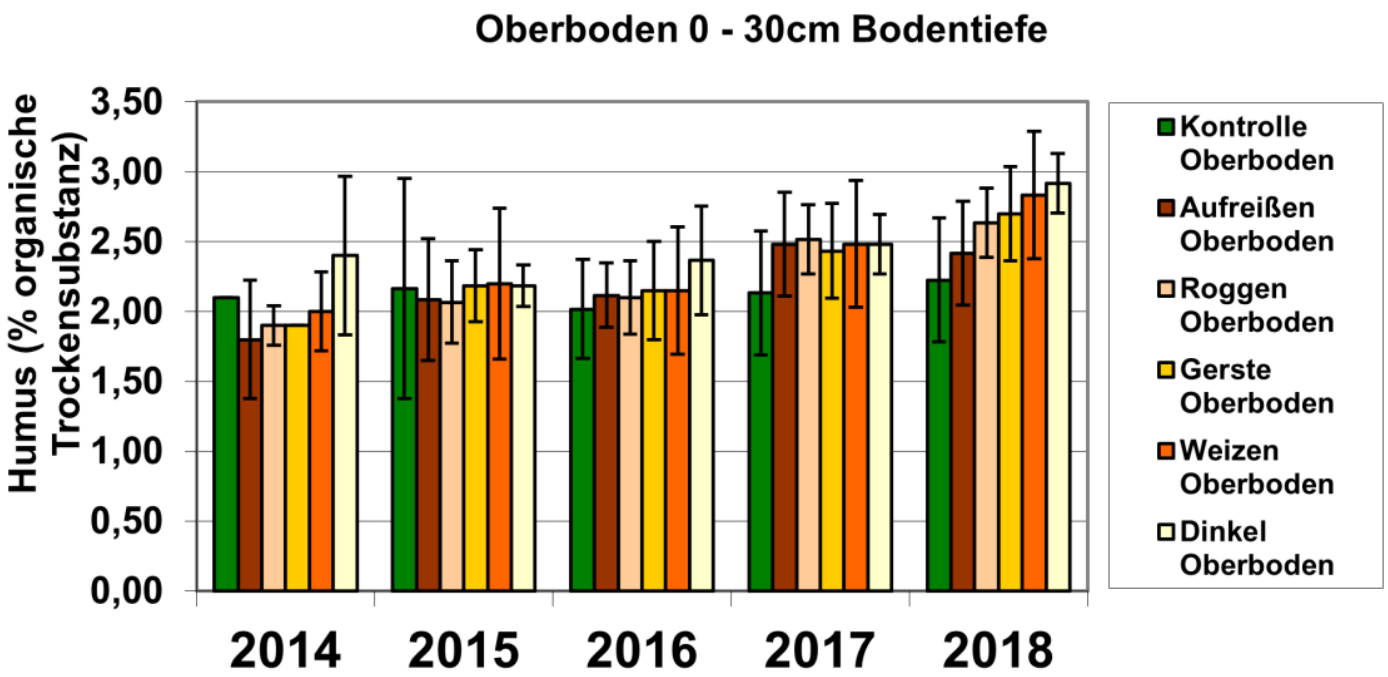

Abb. 4: Organische Substanz (\% organischer Trockensubstanz) des Oberbodens (0-30 cm). // Soil organic matter (\% organic dry matter) of the superficial soil layer $(0-30 \mathrm{~cm})$. 


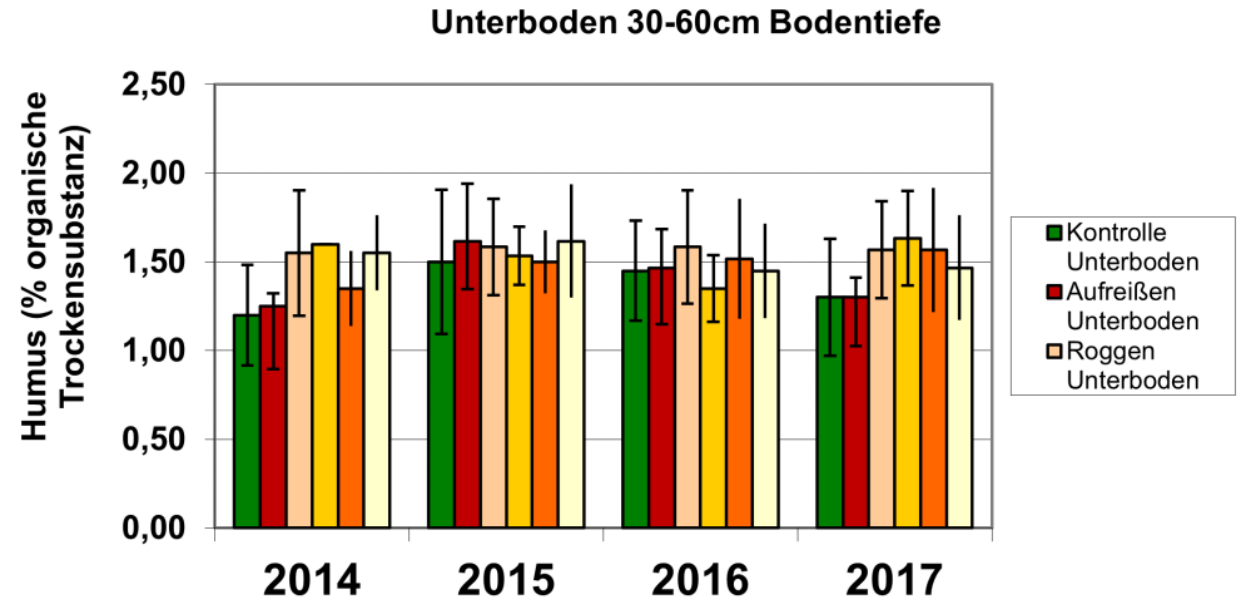

Abb. 5: Organische Substanz (\% organischer Trockensubstanz) des Unterbodens (31-60 cm). // Soil organic matter (\% organic dry matter) of the superficial soil layer $(31-60 \mathrm{~cm})$.
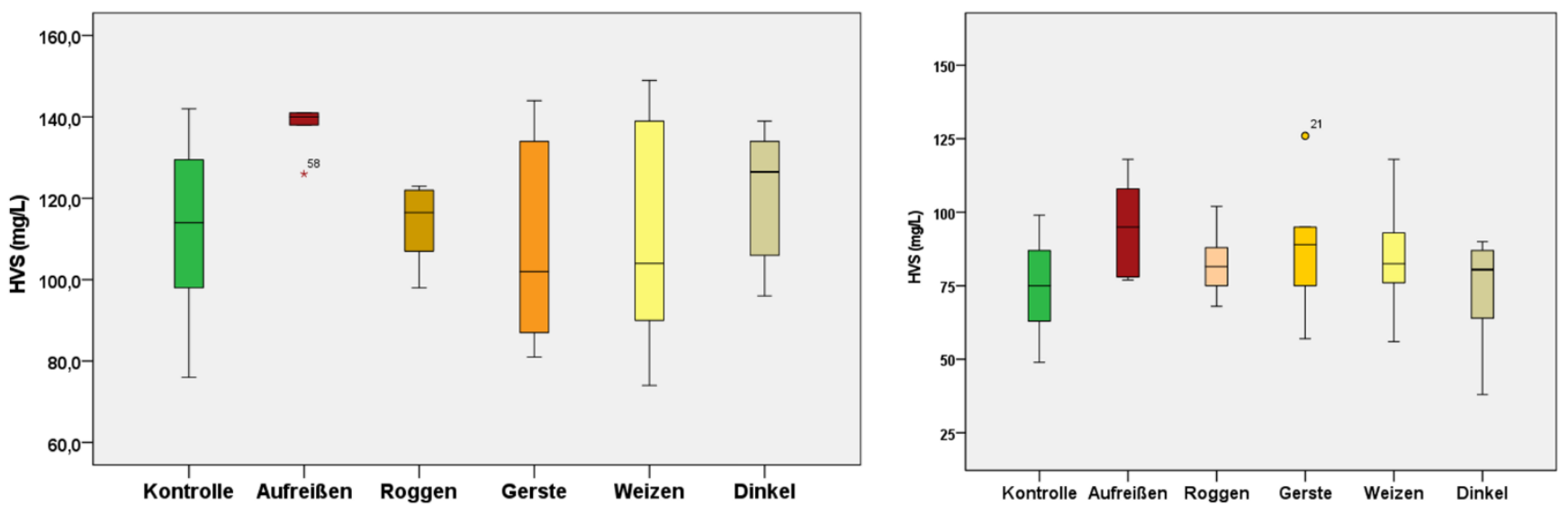

Abb. 6: Gehalt an hefeverwertbarem Stickstoff (mg/l) vor der Lese 2015 (links) und 2018 (rechts) // Concentration of yeast assimilable nitrogen (mg/l) shortly before harvest 2015 (left) and 2028 (right) 


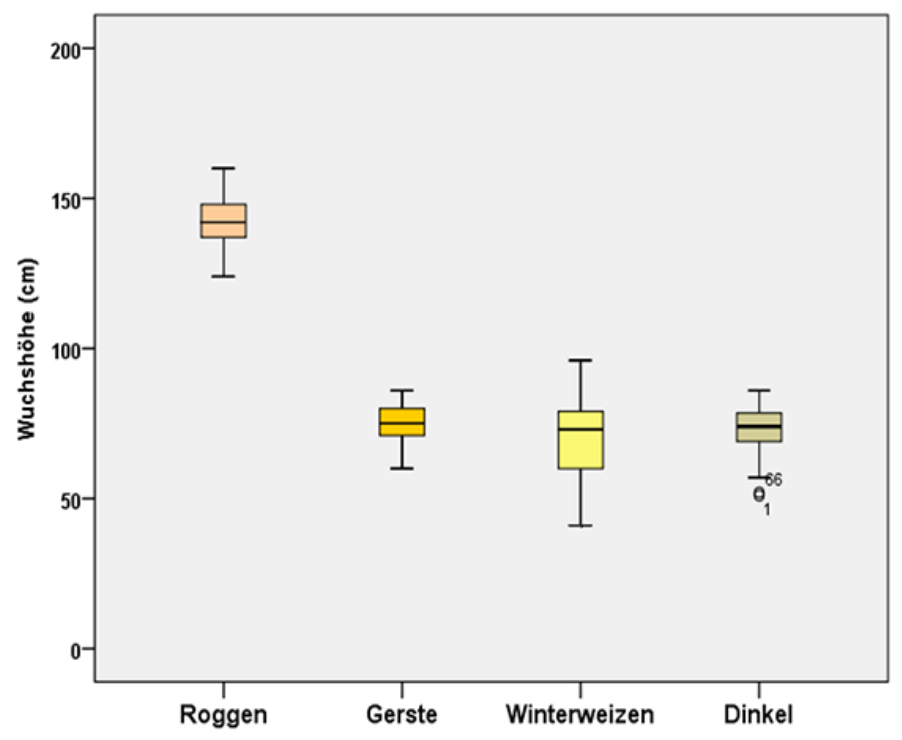

Abb. 7: Wuchshöhe $(\mathrm{cm})$ der Gründüngungspflanzen kurz vor ihrer Blüte. // Growing height $(\mathrm{cm})$ of cover crops shortly before their flowering.
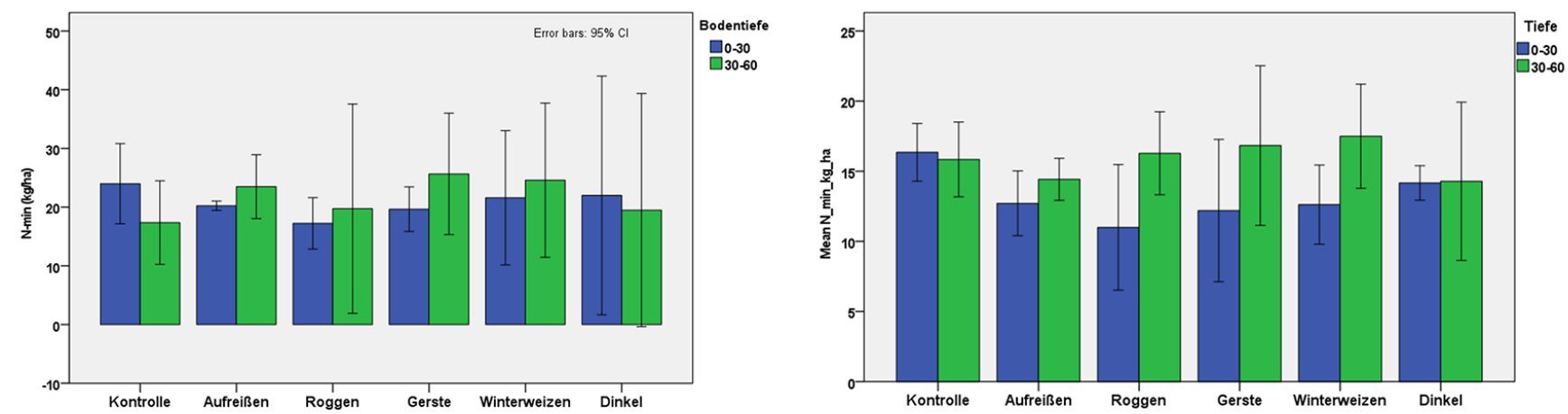

Abb. 8: Freier Stickstoffgehalt N-min (kg/ha) im Sommer 2017 (links) und 2020 (rechts) // Plant available nitrogen (kg/ha) in summer 2017 (left) and 2020 (right). 


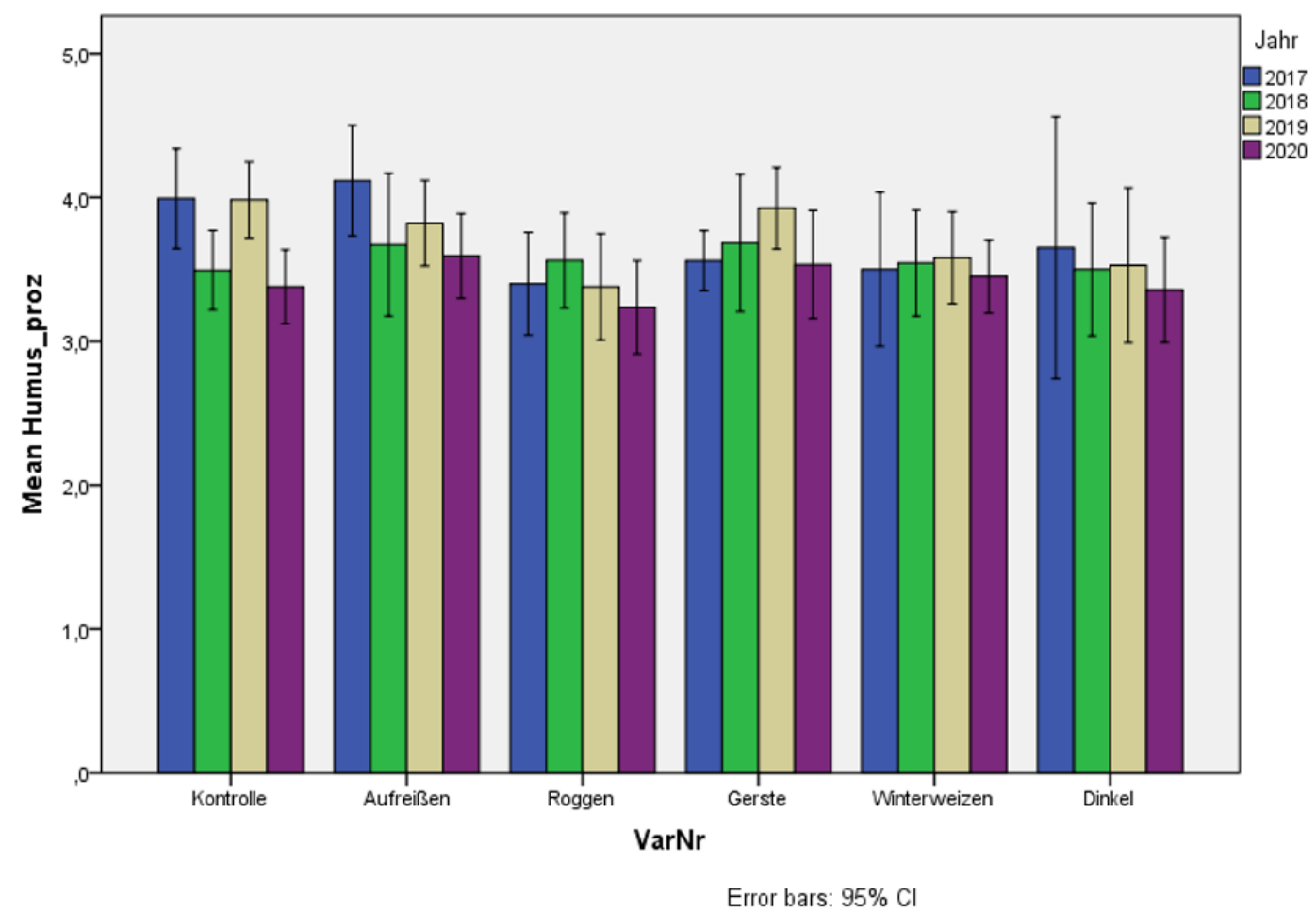

Abb. 9: Mittelwert des Humusgehaltes (\% organischer Trockensubstanz) aus Ober- $(0-30 \mathrm{~cm})$ und Unterboden $(30-60 \mathrm{~cm})$ der vier Versuchsjahre // Mean soil organic matter (\% organic dry matter) of upper $(0-30 \mathrm{~cm})$ and lower $(30-60 \mathrm{~cm})$ soil layer during the four years of the experiment.

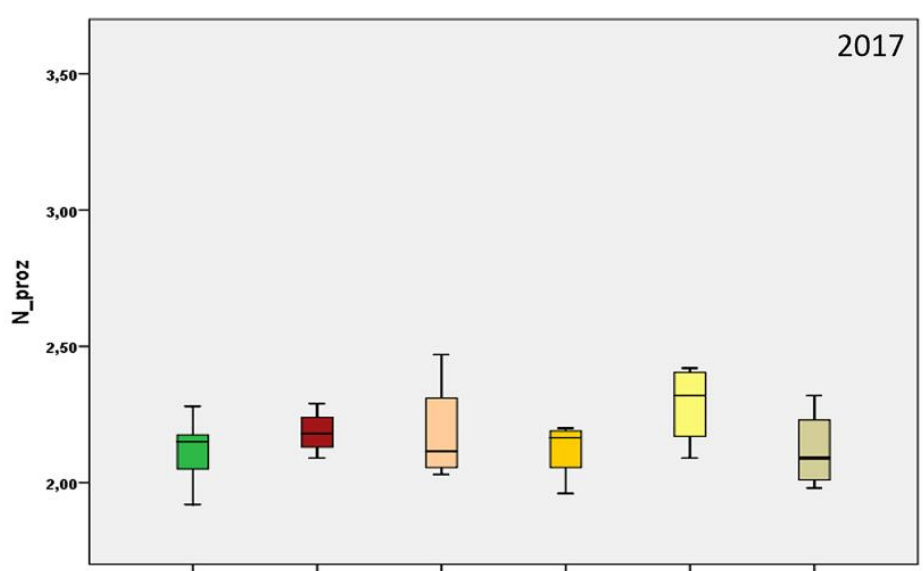

Kontrolle Aufreißen Roggen Gerste Weizen Dinkel

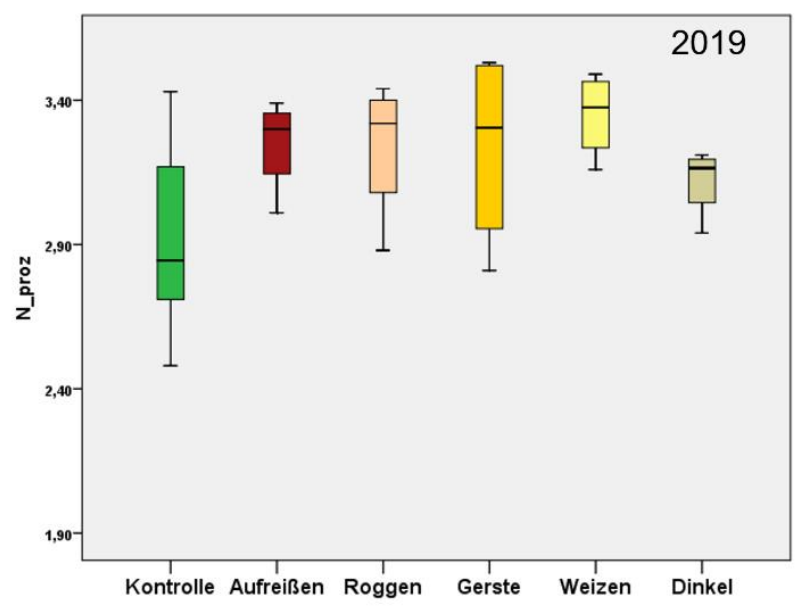

Abb. 10: Stickstoffgehalt (\%N der Trockenmasse) in den Blättern bei Reifebeginn 2017 (links) und 2019 (rechts) // Nitrogen concentration (\% N of dry matter) of the leaves collected at veraison 2017 (left) and 2019 (right). 



Abb. 11: Durchschnittliches Traubengewicht (kg) der Versuchsjahre 2019 (links) und 2020 (rechts) // Mean cluster weight (kg) of the years 2019 (left) and 2020 (right).


Abb. 12: Ertrag (dt)ha) aus den Versuchsvarianten aus den Jahren 2019 (links) und 2020 (rechts) // Yield (decitonne/ha) of the years 2019 (left) and 2020 (right). 

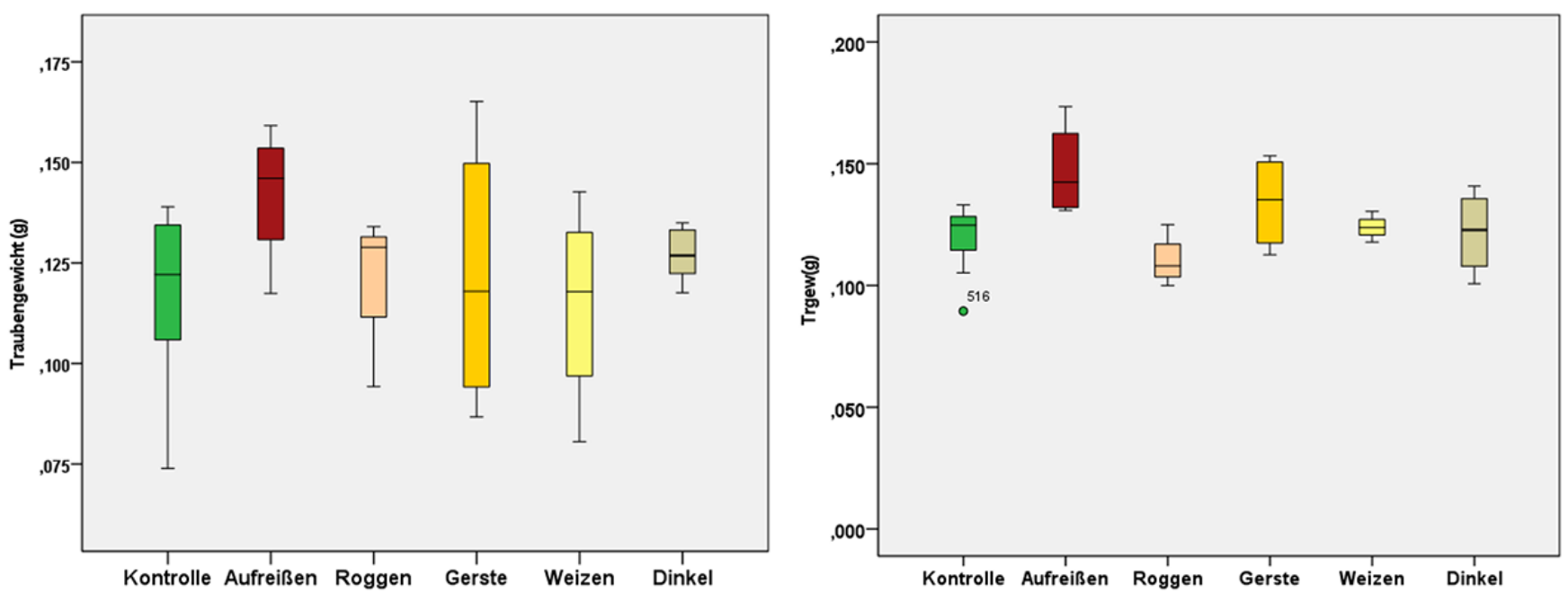

Abb. 13: Schnittholzgewicht (kg/Rebe) für 2019 (links) und (g/Rebe) für 2020 (rechts) // Pruning weight in kg/vine of 2019 left and in (g/vine) for 2020 (right).
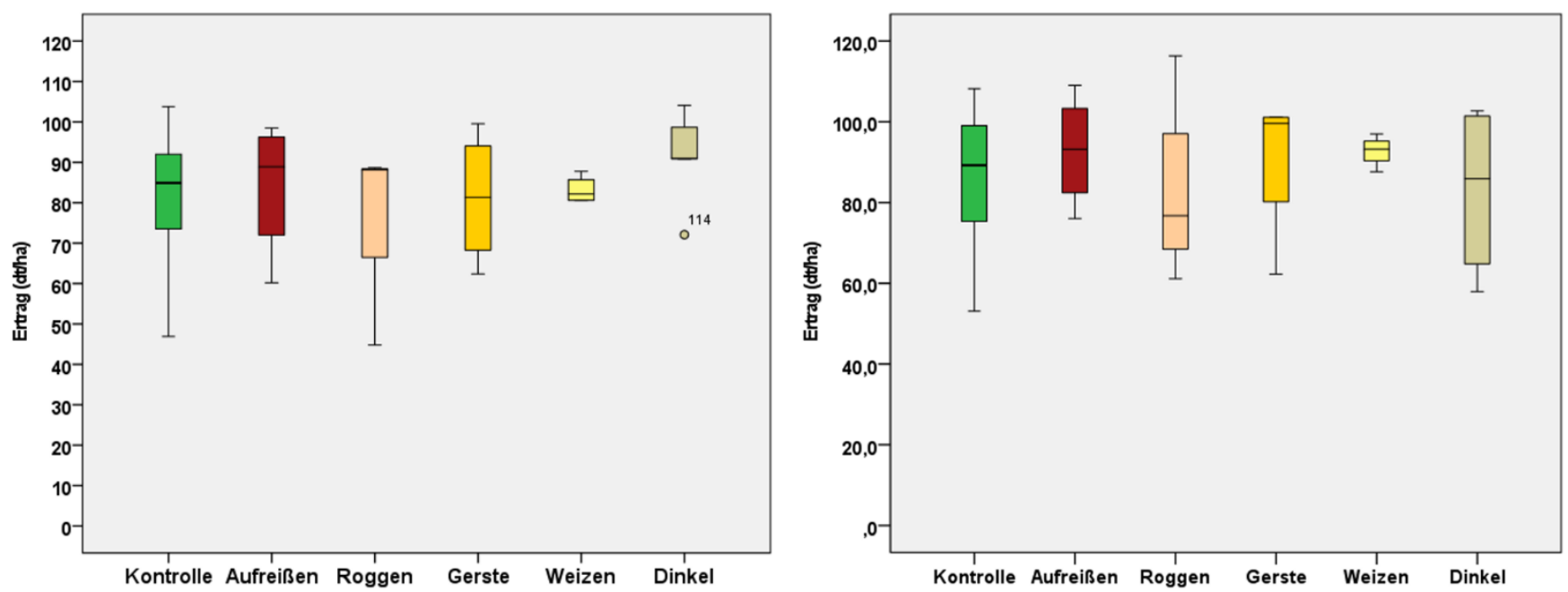

Abb. 14: Ertrag (dt/ha) aus den Versuchsvarianten aus den Jahren 2019 (links) und 2020 (rechts) // Harvest quantity (dt/ha) for each treatment from the years 2019 (left) and 2020 (right).

\section{(c) (i) (9)}

Dieses Werk ist lizenziert unter einer Creative Commons Namensnennung-Nicht kommerziell 4.0 International Lizenz. Quest'opera è distribuita con Licenza Creative Commons Attribuzione - Non commerciale 4.0 Internazionale. This work is licensed under a Creative Commons Attribution-NonCommercial 4.0 International License.

Für alle Abbildungen und Tabellen ohne Nennung des Urhebers gilt: @ Versuchszentrum Laimburg. Per tutte le immagini e tabelle senza menzione dell'artefice vale: (c) Centro di Sperimentazione Laimburg. For all figures and tables without mention of the originator applies: (c) Laimburg Research Centre. 